\title{
Gaussian M-062x/6-31+g (d,p) Calculation of Standard Enthalpy, Entropy and Heat Capacity of Some Fluorinated Alcohol's and Its Radicals at Different Temperatures
}

\author{
Hebah Abdel-Wahab, Joseph Bozzelli \\ Department of Chemistry and Environmental Science, New Jersey Institute of Technology, Newark, New Jersey, United States
}

Email address:

Dr.heathera@gmail.com (H. Abdel-Wahab), jbozelli@njit.edu (J. Bozzelli)

\section{To cite this article:}

Hebah Abdel-Wahab, Joseph Bozzelli. Gaussian M-062x/6-31+g (d,p) Calculation of Standard Enthalpy, Entropy and Heat Capacity of Some Fluorinated Alcohol's and Its Radicals at Different Temperatures. American Journal of Physical Chemistry.

Vol. 9, No. 4, 2020, pp. 101-111. doi: 10.11648/j.ajpc.20200904.13

Received: October 20, 2020; Accepted: December 8, 2020; Published: December 28, 2020

\begin{abstract}
Thermochemical properties of fluorinated alcohols are needed for understanding their stability and reactions in the environment and in thermal process. Structures and thermochemical properties of these species were determined by the Gaussian M-062x/6-31+g (d,p) calculation. Contributions of entropy, $\mathrm{S}^{\circ} 298$, and heat capacities, $\mathrm{Cp}(\mathrm{T}) \mathrm{due}$ to vibration, translation, and external rotation of the molecules were calculated based on the vibration frequencies and structures obtained from the $\mathrm{M}-062 \mathrm{x} / 6-31+\mathrm{g}(\mathrm{d}, \mathrm{p})$ density functional method. Potential barriers are calculated using $\mathrm{M}-062 \mathrm{x} / 6-31+\mathrm{g}(\mathrm{d}, \mathrm{p})$ density functional method and are used to calculate rotor contributions to entropy and heat capacity using integration over energy levels of rotational potential. Enthalpies of formation for 19 fluorinated ethanol and some radicals were calculated with a popular $\mathrm{ab}$ initio and density functional theory methods: the Gaussian M-062x/6-31+g $(\mathrm{d}, \mathrm{p})$ via several series of isodesmic reactions. The recommended ideal gas phase $\Delta \mathrm{Hf}_{2} 98^{\circ}\left(\mathrm{kcal} \mathrm{mol}^{-1}\right)$ values calculated in this study are the following: $-101.74 \pm$ 0.72 for $\mathrm{CH}_{2} \mathrm{FCH}_{2} \mathrm{OH}$; $-113.51 \pm 1.39$ for $\mathrm{CH}_{3} \mathrm{CHFOH}$; $-50.66 \pm 0.75$ for $\mathrm{C} \cdot \mathrm{HFCH}_{2} \mathrm{OH}$; $-56.05 \pm 0.62$ for $\mathrm{CH} \mathrm{FCH}_{2} \cdot \mathrm{OH}$; 45.00 \pm 1.31 for $\mathrm{CH}_{2} \mathrm{FCH}_{2} \mathrm{O} \bullet$; $-59.61 \pm 1.20$ for $\mathrm{CH}_{2} \cdot \mathrm{CHFOH}$; $-67.99 \pm 1.29$ for $\mathrm{CH}_{3} \mathrm{CF} \bullet \mathrm{OH}$; $-58.76 \pm 1.20$ for $\mathrm{CH}_{3} \mathrm{CHFO}$; $154.12 \pm 1.72$ for $\mathrm{CH}_{2} \mathrm{FCHFOH} ;-155.26 \pm 1.67$ for $\mathrm{CF}_{2} \mathrm{HCH}_{2} \mathrm{OH} ;-174.53 \pm 1.54$ for $\mathrm{CH}_{3} \mathrm{CF}_{2} \mathrm{OH}$; $-104.07 \pm 1.45$ for $\mathrm{CH}_{2} \mathrm{FC} \cdot \mathrm{FOH}$; $-105.63 \pm 1.74$ for $\mathrm{C} \cdot \mathrm{HFCFHOH} ;-99.08 \pm 1.57$ for $\mathrm{CH}_{2} \mathrm{FCHFO} \bullet ;-102.34 \pm 1.74$ for $\mathrm{CHF} \mathrm{C}_{2} \cdot \mathrm{HOH} ;-102.23 \pm 1.57$ for $\mathrm{C} \mathrm{F}_{2} \mathrm{CH}_{2} \mathrm{OH} ;-98.86 \pm 1.57$ for $\mathrm{CHF}_{2} \mathrm{CH}_{2} \mathrm{O} \bullet ;-119.41 \pm 1.74$ for $\mathrm{CH}_{2} \cdot \mathrm{CF}_{2} \mathrm{OH} ;-110.56 \pm 1.62$ for $\mathrm{CH}_{3} \mathrm{CF}_{2} \mathrm{O} \bullet$. Entropies $\left(\mathrm{S}_{2} 98^{\circ}\right.$ in cal $\mathrm{mol}^{-1} \mathrm{~K}^{-1}$ ) were estimated using the $\mathrm{M}-062 \mathrm{x} / 6-31+\mathrm{g}(\mathrm{d}, \mathrm{p})$ computed frequencies and geometries. Rotational barriers were determined and hindered internal rotational contributions for $\mathrm{S} 298^{\circ}-1500^{\circ}$, and $\mathrm{Cp}(\mathrm{T})$ were calculated using the rigid rotor harmonic oscillator approximation, with direct integration over energy levels of the intramolecular rotation potential energy curves.
\end{abstract}

Keywords: Computation, Gaussian, Thermochemical, Enthalpy, Entropy

\section{Introduction}

Fluorinated hydrocarbons used as refrigerants, in polymers, heat exchange fluids, and as solvents. They are present in the atmosphere, lithosphere, and hydrosphere. Because of their less adverse effects on the stratospheric ozone layer, they are used in place of greenhouse gases. [1] Fluorinated hydrocarbons exist as compounds ranging from pure to oxidized intermediates resulting from oxidation in the environment. In order to study their reactivity in biological systems, lifetimes, and in the environment, it's critical to understand the chemical and thermodynamic properties of fluorocarbons and their breakdown intermediates.

The thermochemistry of fluorinated alcohols with one carbon atom were studied in the past and are in the literature [2]. In 2016 Hang Wang studied thermodynamic properties of fluorinated methanol using CBS-QB3, M06, M06-2X, WB97X, W1U, B3LYP, CBS-APNO and G4 Calculations. Small standard deviation suggests good error cancellation of 
work reactions and accuracy. M06-2x/6-31+g (d,p) calculation have small values for standard deviations, it is an accurate method to calculate Enthalpy of fluorinated alcohols, it shows the second smallest standard deviation after CBS-QB3 method of calculation

Halogenated compounds are highly stable, have low reactivity and are valued chemicals in industry [3]. Due to its widespread use and their persistence in the environment, they are of concern to the environment. In order to understand the oxidation and reduction reactions involving such molecules, their thermochemical properties must be studied. [3]

\section{Computational Method}

Composite calculations and series of Isodesmic Reactions are used to calculate enthalpy of formation of fluorinated ethanols. All calculations are performed using the Gaussian 16 program. The DFT method M06-2x is used to initially analyze optimized structures, frequencies, thermo energies and internal rotors of the molecules studied It's a Global-hybrid meta-GGA density functional approximation, GGA, generalized gradient approximation, in which the density functional depends on the up and down spin densities and their reduced gradient, meta GGA, in which the functional also depends on the up and down spin kinetic energy densities, hybrid GGA, a combination of GGA with Hartree-Fock exchange, hybrid meta GGA, a combination of meta GGA with Hartree-Fock exchange [21]. All reported values are for standard state of $298 \mathrm{~K}$ and 1 atm. We continue the calculation of fluorinated alcohols in this study with this method, because the M-062x/6-31+g (d,p) level of calculation have been applied to fluoro hydrocarbons [4] with small reported standard deviations values.

\section{Isodesmic and Isogyric Reaction}

The enthalpy of formation of mono and di fluorinated ethanol's and its radicals has been calculated using Gaussian M-062x/6-31+g (d,p) method of calculation. In order to calculate the enthalpy of formation of fluorinated ethanols using this method, we use the calculated enthalpies of formation in work reactions along with reference species. The number of each type of bond must be conserved in the isodesmic reactions in order to cancel any systematic error in the molecular orbital calculations using this method. Calculations of enthalpies of formation is allowed to accuracies close to experimental values by the careful choice of the isodesmic reactions [5]. Taking 1fluoroethanol as an example, two isodesmic reactions (Table 2) are selected to determine the $\Delta \mathrm{Hf} 298$ of the target molecule, 1-fluoroethanol. Since the $\Delta \mathrm{Hf} 298$ values of all species but 1-fluoroethanol in 1- 2 (Table 2) are known, the $\Delta \mathrm{Hf} 298$ of the target species 1-fluoroethanol, is obtained from this data and the calculated $\Delta H r x n, 298 . \Delta H f 298$ calculated using two different reference molecules are within $\pm .2 \mathrm{Kcal} \mathrm{mol}^{-1}$.

\section{Reference Species}

Standard enthalpy of formation for the reference species used in the isodesmic work reactions along with their uncertainties are listed, table 1. Table 2 provides one example on the method of Isodesmic Work Reactions used for the calculation of the Standard Enthalpy of Formation $\Delta_{\mathrm{f}} \mathrm{H}_{2} 98$ for the fluoroethanols.

Table 1. Reference Species in the Isodesmic Reactions Standard Enthalpy of Formation Values (kcal mol ${ }^{-1}$ [6]).

\begin{tabular}{|c|c|c|c|}
\hline Species & $\overline{\Delta_{\mathrm{f}} \mathrm{H}^{\mathrm{O}}{ }_{(298)}}$ & Species & $\overline{\Delta_{\mathrm{f}} \mathbf{H}^{\mathrm{O}}{ }_{(298)}}$ \\
\hline \multirow{2}{*}{$\mathrm{CH}_{3} \mathrm{~F}$} & $-56.54 \pm 0.07^{\mathrm{a}}$ & $\mathrm{CH}_{3} \mathrm{OOH}$ & $-30.96 \pm 0.67^{b}$ \\
\hline & $-56.62 \pm 0.48^{\mathrm{h}}$ & $\mathrm{CH}_{3} \mathrm{CH}_{2} \mathrm{OOH}$ & $-38.94 \pm 0.81^{b}$ \\
\hline $\mathrm{CH}_{3} \mathrm{CH}_{2} \mathrm{~F}$ & $-65.42 \pm 1.11^{\mathrm{a}}$ & $\mathrm{CH}_{3} \mathrm{CH}_{2} \mathrm{CH}_{2} \mathrm{OOH}$ & $-44.03 \pm 0.67^{b}$ \\
\hline $\mathrm{CH}_{3} \mathrm{CH}_{2} \mathrm{CH}_{2} \mathrm{~F}$ & $-70.24 \pm 1.30^{\mathrm{a}}$ & $\mathrm{CH}_{3} \mathrm{OO}^{\circ}$ & $2.37 \pm 1.24^{\mathrm{b}}$ \\
\hline $\mathrm{CH}_{\mathrm{F}}$ & $-108.07 \pm 1.46^{\mathrm{a}}$ & CHCHOD.CHCHCHOD. & $-6.19 \pm 0.92^{b}$ \\
\hline $\mathrm{CH}_{2} \mathrm{H}_{2}$ & $-107.67 \pm 0.48^{\mathrm{h}}$ & $\mathrm{CH}_{3} \mathrm{CH}_{2} \mathrm{OO} \mathrm{CH}_{3} \mathrm{CH}_{2} \mathrm{CH}_{2} \mathrm{OO}$ & $-11.35 \pm 1.24^{b}$ \\
\hline $\mathrm{CH}_{3} \mathrm{CHF}_{2}$ & $-120.87 \pm 1.62^{\mathrm{a}}$ & $\mathrm{CH}_{4}$ & $-17.81 \pm 0.01^{\mathrm{c}}$ \\
\hline $\mathrm{CH}_{3} \mathrm{CH}_{2} \mathrm{CHF}_{2}$ & $-125.82 \pm 1.65^{\mathrm{a}}$ & $\mathrm{CH}_{3} \mathrm{CH}_{3}$ & $-20.05 \pm 0.04^{\mathrm{c}}$ \\
\hline $\mathrm{CHF}_{3}$ & $\begin{array}{l}-166.71 \pm 1.97^{\mathrm{h}} \\
-166.09 \pm 0.48^{\mathrm{h}}\end{array}$ & $\mathrm{CH}_{3} \mathrm{CH}_{2} \mathrm{CH}_{3} \mathrm{CH}_{3} \mathrm{CH}_{2} \mathrm{CH}_{2} \mathrm{CH}_{3}$ & $\begin{array}{l}-25.01 \pm 0.06^{\mathrm{i}} \\
-30.07+0.08^{\mathrm{i}}\end{array}$ \\
\hline $\mathrm{CH}_{3} \mathrm{CF}_{3}$ & $-180.51 \pm 2.05^{\mathrm{a}}$ & $\mathrm{CH}_{3} \mathrm{O}^{-}$ & $5.15 \pm 0.08^{c}$ \\
\hline $\mathrm{CH}_{3} \mathrm{CH}_{2} \mathrm{CF}_{3}$ & $-185.48 \pm 2.15^{\mathrm{a}}$ & $\mathrm{CH}_{3} \mathrm{CH}_{2} \mathrm{O}^{-}$ & $-3.01^{\mathrm{d}}$ \\
\hline $\mathrm{CH}_{3} \cdot$ & $34.98 \pm 0.02^{\mathrm{c}}$ & $\mathrm{OH}$ & $8.96 \pm 0.01^{\mathrm{c}}$ \\
\hline $\mathrm{CH}_{3} \mathrm{CH}_{2} \cdot$ & $28.65 \pm 0.07^{\mathrm{c}}$ & $\mathrm{CH}_{3} \mathrm{OH}$ & $-47.97 \pm 0.04^{\mathrm{c}}$ \\
\hline $\mathrm{CH}_{3} \mathrm{CH}_{2} \mathrm{CH}_{2}{ }^{\circ}$ & $\begin{array}{l}24.21 \pm 0.24^{\mathrm{gi}} \\
24.18^{\mathrm{i}}\end{array}$ & $\mathrm{CH}_{3} \mathrm{CH}_{2} \mathrm{OH}$ & $-56.07 \pm 0.05^{\mathrm{i}}$ \\
\hline $\mathrm{H}$ & $52.10^{\mathrm{c}}$ & $\mathrm{HOO}^{\circ}$ & $2.94^{\mathrm{cj}}$ \\
\hline $\mathrm{O}$ & $59.57^{\mathrm{c}}$ & $\mathrm{HOOH}$ & $\begin{array}{l}-32.39 \pm 0.04^{\mathrm{fj}} \\
-32.37^{\mathrm{i}}\end{array}$ \\
\hline
\end{tabular}

aWang [2], bWang [6], cRuscic [7], dBurke [8], eChase [9], fLuo [10], gBodi [11], hPedley, iATcT Tables [12], hCsontos [13]. jThe value we used in this study [6] 
Table 2. Example for Enthalpy of Formation Calculations for 1-fluoroethanol using Isodesmic Reactions; using different reference molecules, Units in kcal $\mathrm{mol}^{-1}$

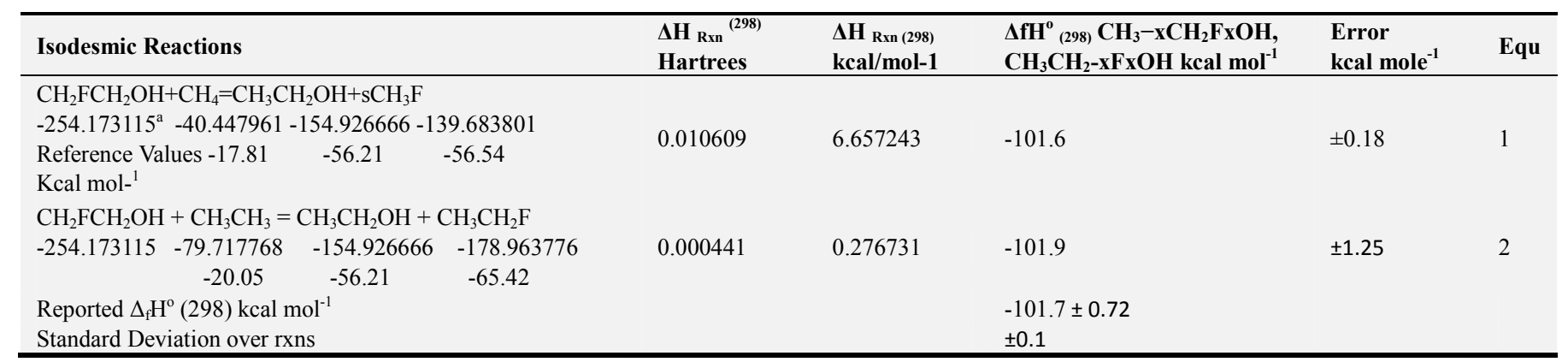

a Hartrees, $\mathrm{kcal} \mathrm{mole}^{-1} * \mathrm{SD}$ Standard Deviation $\mathrm{kcal} \mathrm{mol}^{-1}$ Errors reported avg of sum of uncertainties in rxn's reference species

\section{Results and Discussion}

Optimized Structures

Table 3. Optimized geometries showing bond angles and bond distances for target fluorinated ethanol and their related radicals calculated by M06-2x/6$31+g(d, p)$ level of theory. Bond lengths in A, bond angles in degree, dihedral angle in degree.
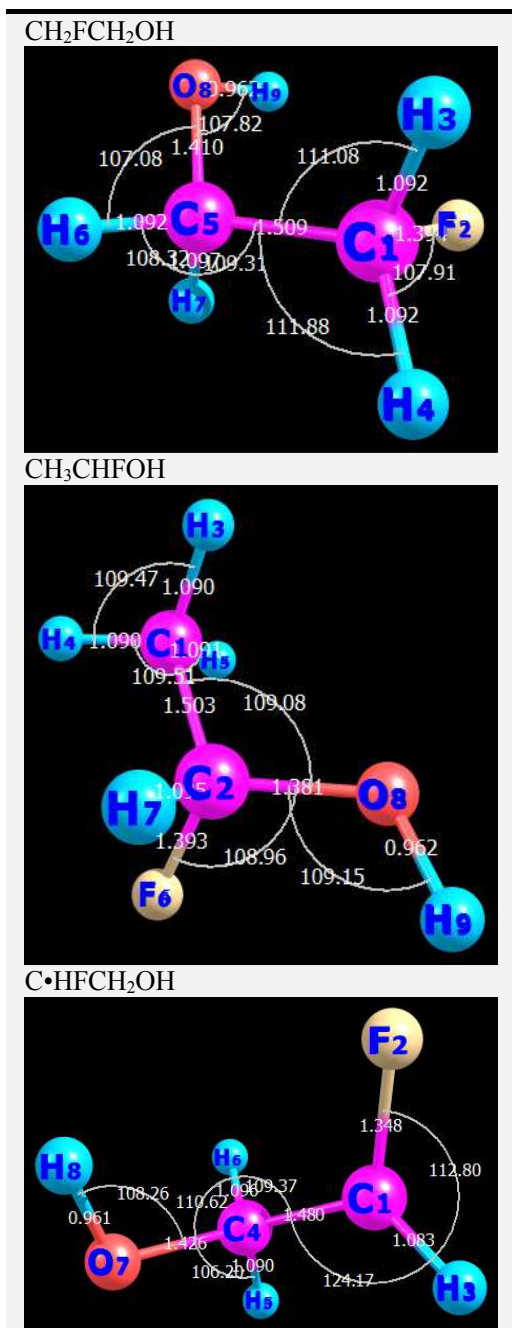

$\mathrm{CH}_{2} \mathrm{FCH} \cdot \mathrm{OH}$
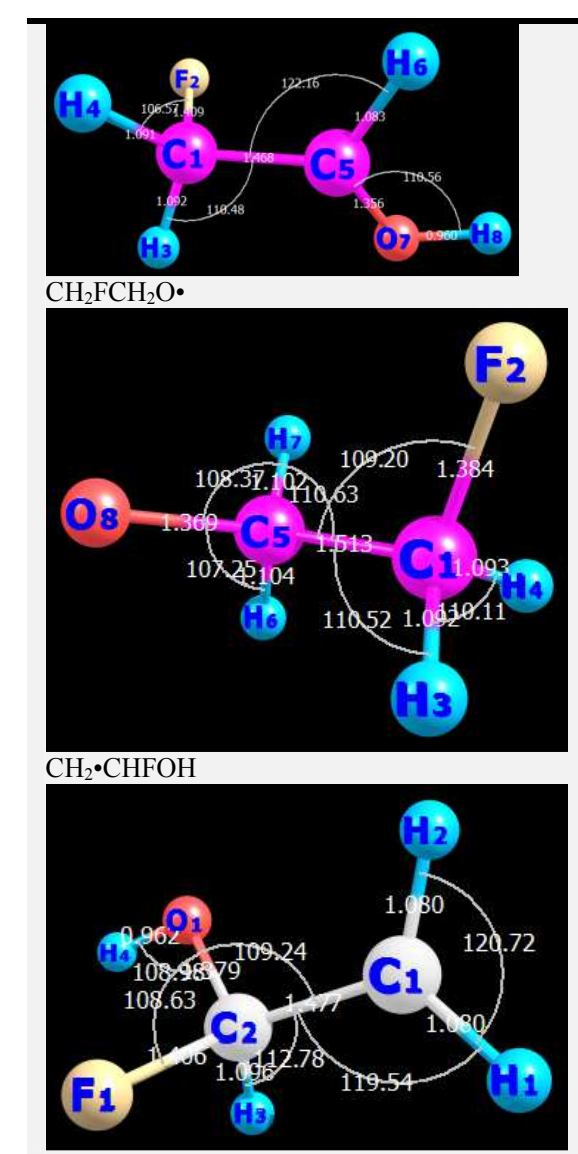

$\mathrm{CH}_{3} \mathrm{CF} \bullet \mathrm{OH}$

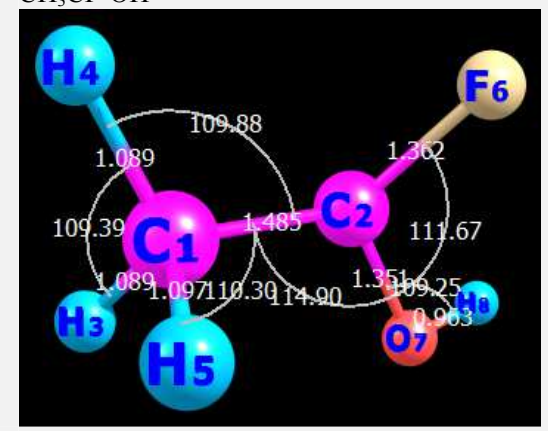

$\mathrm{CH}_{3} \mathrm{CHFO} \cdot$ 

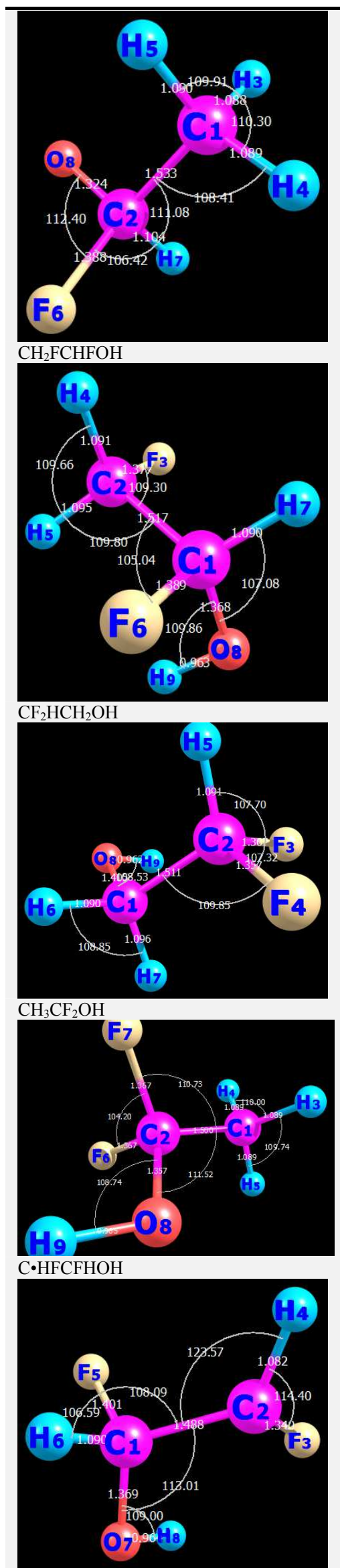

$\mathrm{CH}_{2} \mathrm{FC} \cdot \mathrm{FOH}$
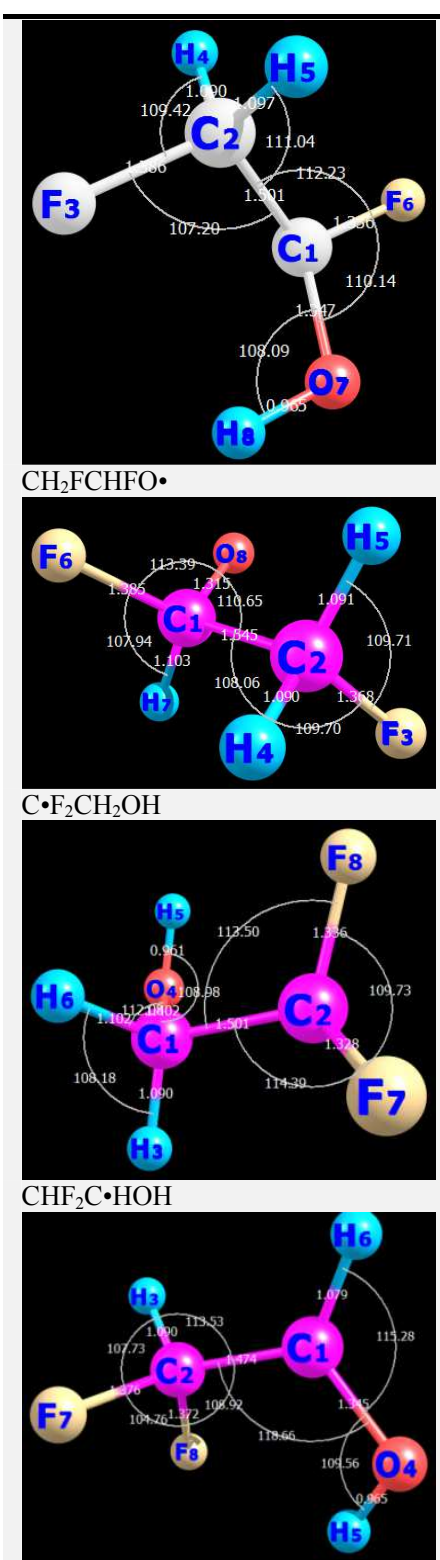

$\mathrm{CHF}_{2} \mathrm{CH}_{2} \mathrm{O} \bullet$
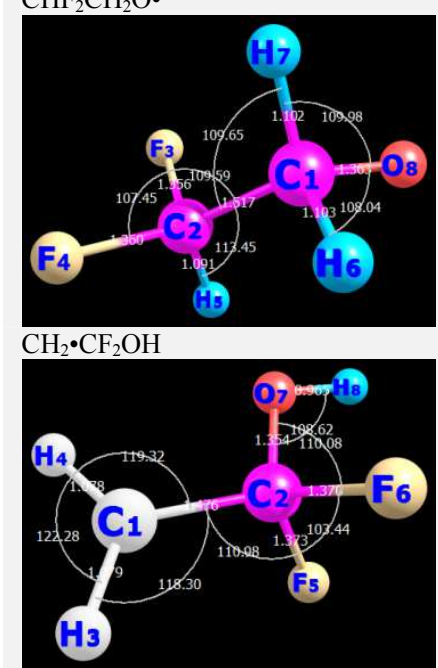

$\mathrm{CH}_{3} \mathrm{CF}_{2} \mathrm{O} \cdot$ 


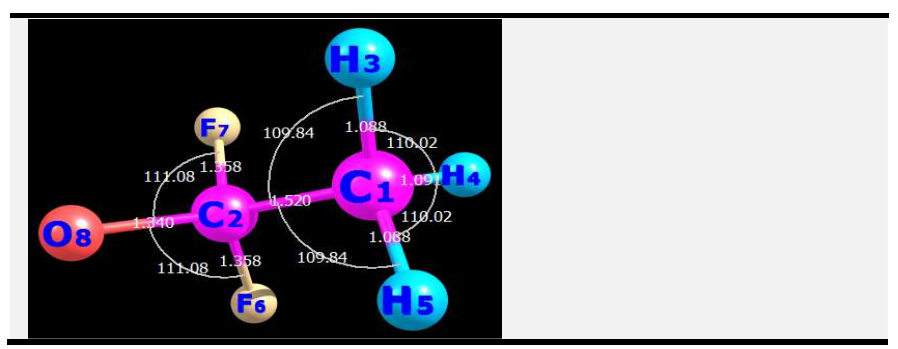

\section{Standard Enthalpy Values}

Table 4. Standard Enthalpy of Formation using isodesmic reactions: Monofluoro and Difluoro- Ethanols using the M06-2x/6-31+g (d,p) Level of Theory.

Errors reported as sum of avg uncertainty in rxn's reference specie.

\begin{tabular}{|c|c|c|c|c|}
\hline Isodesmic Reactions arget Specie & $\Delta H_{\text {Rxn (298) }}$ Hartrees & $\Delta \mathrm{H}_{\mathrm{Rxn}(298)} \mathrm{Kcal} / \mathrm{mole}^{1}$ & $\Delta_{\mathrm{f}} \mathrm{H}_{(298)} \mathrm{kcal} \mathrm{mol}^{-1}$ & Error kcal mol ${ }^{-1}$ \\
\hline \multicolumn{5}{|l|}{$\mathrm{CH}_{2} \mathrm{FCH}_{2} \mathrm{OH}+\mathrm{CH}_{4}=\mathrm{CH}_{3} \mathrm{CH}_{2} \mathrm{OH}+\mathrm{CH}_{3} \mathrm{~F}$} \\
\hline$-254.173115-40.447961-154.926666-139.683801$ & 0.010609 & 6.657243 & -101.6 & \pm 0.18 \\
\hline $\begin{array}{lll}-17.81 & -56.21 & -56.54\end{array}$ & & & & \\
\hline \\
\hline$-254.173115-79.717768-154.926666-178.963776$ & 0.000441 & 0.276731 & -101.9 & \pm 1.25 \\
\hline \multicolumn{5}{|l|}{$\begin{array}{lll}-20.05 & -56.21 & -65.42\end{array}$} \\
\hline \multirow{4}{*}{$\begin{array}{l}\text { Reported } \Delta_{\mathrm{f}} \mathrm{H}^{\mathrm{o}}(298) \mathrm{kcal} \mathrm{mol}^{-1} \\
\text { Standard Deviation over rxns } \\
\mathrm{CH}_{3} \mathrm{CHFOH}+\mathrm{CH}_{3} \mathrm{CH}_{2} \mathrm{CH}_{3}=\mathrm{CH}_{3} \mathrm{CH}_{2} \mathrm{OH}+\mathrm{CH}_{3} \mathrm{CH}_{2} \mathrm{CH}_{2} \mathrm{~F} \\
-254.19203-118.990915-154.926666-218.237381\end{array}$} & & & \multicolumn{2}{|l|}{$-101.7 \pm 0.72$} \\
\hline & & & \pm 0.1 & \\
\hline & & & & \\
\hline & 0.018898 & 11.85867 & -113.3 & \pm 1.52 \\
\hline $\begin{array}{lll}-25.02 & -56.21 & -70.24\end{array}$ & & & & \\
\hline \multicolumn{5}{|l|}{$\mathrm{CH}_{3} \mathrm{CHFOH}+\mathrm{CH}_{3} \mathrm{CH}_{3}=\mathrm{CH}_{3} \mathrm{CH}_{2} \mathrm{OH}+\mathrm{CH}_{3} \mathrm{CH}_{2} \mathrm{~F}$} \\
\hline$-254.19203-79.717768-154.926666-178.963776$ & 0.019356 & 12.14606 & -113.7 & \pm 1.25 \\
\hline $\begin{array}{lll}-20.05 & -56.21 & -65.42\end{array}$ & & & & \\
\hline Reported $\Delta_{\mathrm{f}} \mathrm{H}^{\mathrm{o}}(298) \mathrm{kcal} \mathrm{mol}^{-1}$ & & & $-113.5 \pm 1.39$ & \\
\hline Standard Deviation over rxns & & & \pm 0.2 & \\
\hline \multicolumn{5}{|l|}{$\mathrm{C} \cdot \mathrm{HFCH}_{2} \mathrm{OH}+\mathrm{CH}_{3} \mathrm{CH}_{2} \mathrm{CH}_{3}=\mathrm{CH}_{3} \mathrm{CH}_{2} \mathrm{O} \cdot+\mathrm{CH}_{3} \mathrm{CH}_{2} \mathrm{CH}_{2} \mathrm{~F}$} \\
\hline$-253.518298-118.990915-154.268107-218.237381$ & 0.003725 & 2.337471 & -50.6 & \pm 1.42 \\
\hline $\begin{array}{lll}-25.02 & -3.01 & -70.24\end{array}$ & & & & \\
\hline \multicolumn{5}{|l|}{$\mathrm{C} \cdot \mathrm{HFCH}_{2} \mathrm{OH}+\mathrm{CH}_{4}=\mathrm{CH}_{3} \mathrm{CH}_{2} \mathrm{O} \cdot+\mathrm{CH}_{3} \mathrm{~F}$} \\
\hline$-253.518298-40.447961-154.268107-139.683801$ & 0.014351 & 9.005382 & -50.8 & \pm 0.08 \\
\hline $\begin{array}{lll}-17.81 & -3.01 & -56.54\end{array}$ & & & & \\
\hline Reported $\Delta_{\mathrm{f}} \mathrm{H}^{\mathrm{o}}(298) \mathrm{kcal} \mathrm{mol}^{-1}$ & & & $-50.7 \pm 0.75$ & \\
\hline Standard Deviation over rxns & & & \pm 0.09 & \\
\hline \multicolumn{5}{|l|}{$\mathrm{CH}_{2} \mathrm{FCH} \cdot \mathrm{OH}+\mathrm{CH}_{3} \mathrm{CH}_{3}=\mathrm{CH}_{3} \mathrm{CH}_{2} \mathrm{O} \bullet+\mathrm{CH}_{3} \mathrm{CH}_{2} \mathrm{~F}$} \\
\hline $\begin{array}{l}-253.518298-79.717768-154.268107-178.963776 \\
-20.05-3.01-65.42\end{array}$ & 0.012436 & 7.803702 & -56.2 & \pm 1.15 \\
\hline \multicolumn{5}{|l|}{$\mathrm{CH}_{2} \mathrm{FCH} \bullet \mathrm{OH}+\mathrm{CH}_{4}=\mathrm{CH}_{3} \mathrm{CH}_{2} \mathrm{O} \bullet+\mathrm{CH}_{3} \mathrm{~F}$} \\
\hline$-253.518298-40.447961-154.268107-139.683801$ & 0.022604 & 14.18421 & -55.9 & \pm 0.08 \\
\hline \multirow{3}{*}{$\begin{array}{l}\quad-17.81 \quad-3.01 \\
\text { Reported } \Delta_{\mathrm{f}} \mathrm{H}^{\mathrm{o}}(298) \mathrm{kcal} \mathrm{mol}^{-1} \\
\text { Standard Deviation over rxns }\end{array}$} & & & & \\
\hline & & & $-56.1 \pm 0.62$ & \\
\hline & & & \pm 0.1 & \\
\hline \multicolumn{5}{|l|}{$\mathrm{CH}_{2} \mathrm{FCH}_{2} \mathrm{O} \bullet+\mathrm{CH}_{3} \mathrm{CH}_{2} \mathrm{CH}_{3}=\mathrm{CH}_{3} \mathrm{CHO} \bullet+\mathrm{CH}_{3} \mathrm{CH}_{2} \mathrm{CH}_{2} \mathrm{~F}$} \\
\hline $\begin{array}{cccc}-253.509195 & -118.990915 & -154.268107 & -218.237381 \\
& -25.02 & -3.01 & -70.24\end{array}$ & -0.00538 & -3.37474 & -44.9 & \pm 1.42 \\
\hline \multicolumn{5}{|l|}{$\mathrm{CH}_{2} \mathrm{FCH}_{2} \mathrm{O} \bullet+\mathrm{CH}_{4}=\mathrm{CH}_{3} \mathrm{O} \bullet+\mathrm{CH}_{3} \mathrm{CH}_{2} \mathrm{~F}$} \\
\hline$-253.509195-40.447961-114.989112-178.963776$ & 0.004268 & 2.678208 & -45.1 & \pm 1.20 \\
\hline $\begin{array}{lll}-17.81 & 5.15 & -65.42\end{array}$ & & & & \\
\hline Reported $\Delta_{\mathrm{f}} \mathrm{H}^{\mathrm{o}}(298) \mathrm{kcal} \mathrm{mol}^{-1}$ & & & $-45.0 \pm 1.31$ & \\
\hline Standard Deviation over rxns & & & \pm 0.1 & \\
\hline $\mathrm{CH}_{2} \cdot \mathrm{CHFOH}+\mathrm{CH}_{4}=\mathrm{CH}_{3} \mathrm{O} \cdot+\mathrm{CH}_{3} \mathrm{CH}_{2} \mathrm{~F}$ & & & & \\
\hline$-253.532134-40.447961-114.989112-178.963776$ & 0.027207 & 17.07264 & -59.5 & \pm 1.2 \\
\hline $\begin{array}{lll}-17.81 & 5.15 & -65.42\end{array}$ & & & & \\
\hline $\mathrm{CH}_{2} \bullet \mathrm{CHFOH}+\mathrm{CH}_{3} \mathrm{CH}_{3}=\mathrm{CH}_{3} \mathrm{CH}_{2} \mathrm{O} \bullet+\mathrm{CH}_{3} \mathrm{CH}_{2} \mathrm{~F}$ & & & & \\
\hline $\begin{array}{cccc}-253.532134 & -79.717768 & -154.268107 & -178.963776 \\
& -20.05 & -3.01 & -65.42\end{array}$ & 0.018019 & 11.30708 & -59.7 & \pm 1.15 \\
\hline Reported $\Delta_{\mathrm{f}} \mathrm{H}^{\mathrm{o}}(298) \mathrm{kcal} \mathrm{mol}^{-1}$ & & & $-59.6 \pm 1.20$ & \\
\hline Standard Deviation over rxns & & & \pm 0.1 & \\
\hline
\end{tabular}

Enthalpies of formation were determined from isodesmic work reactions from $\mathrm{M}-062 \mathrm{x} / 6-31+\mathrm{g}(\mathrm{d}, \mathrm{p})$ method of calculation. The standard enthalpy of formation for the reference species along with their uncertainties, which are used the isodesmic work reactions, table 4, are listed in table 1 in $\mathrm{kcal} \mathrm{mol}^{-1}$. The standard deviation was calculated [14] for all Enthalpies of formation values for all 19 fluorinated ethanol and are included in table 4. Details of the method of standard deviation [14] and example calculation(s) are shown in the *Supporting Information table provided. 


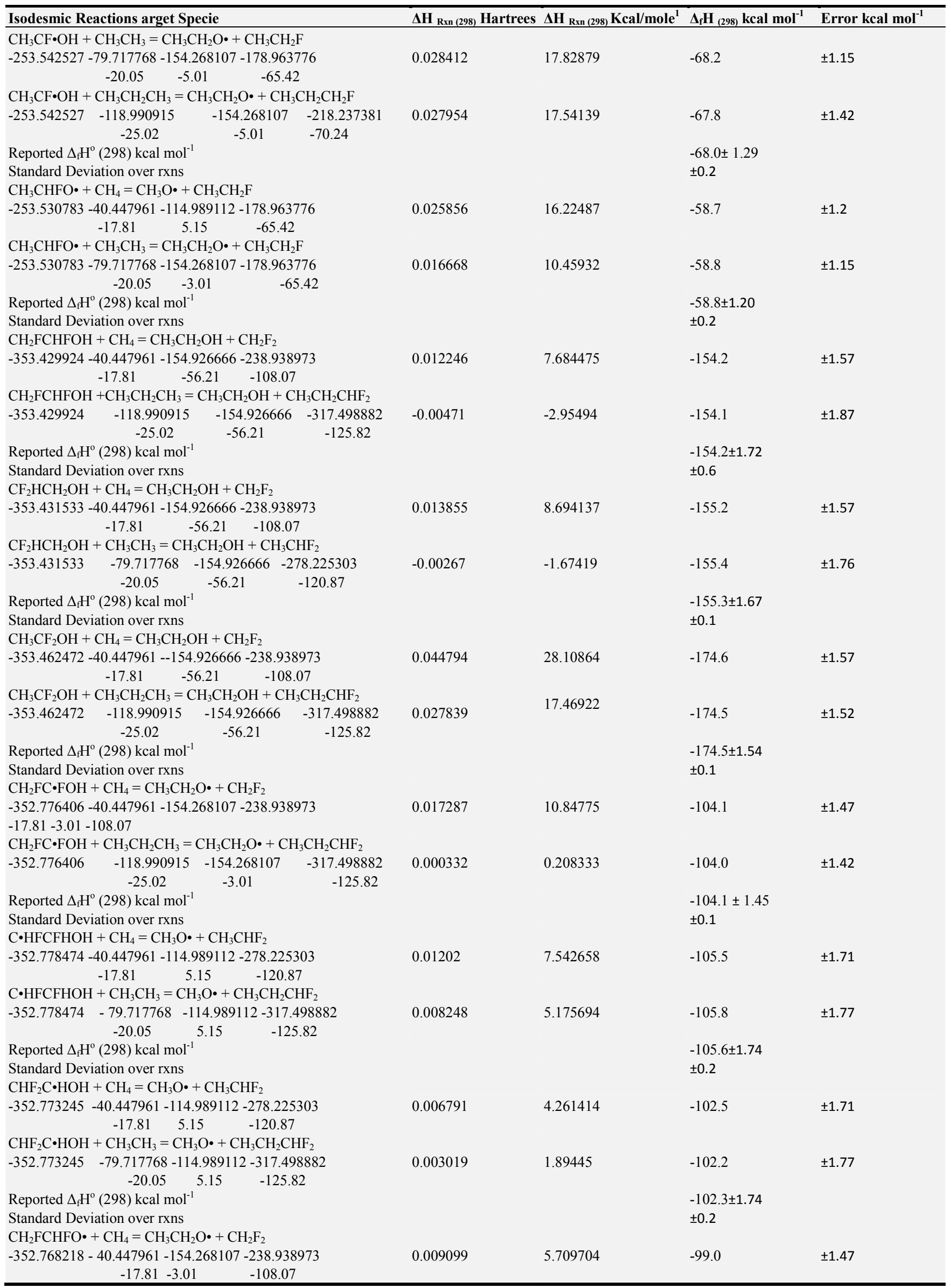




\begin{tabular}{|c|c|c|c|c|}
\hline Isodesmic Reactions arget Specie & $\Delta H_{\text {Rxn (298) }}$ Hartrees & $\Delta \mathrm{H}_{\mathrm{Rxn}(298)} \mathrm{Kcal} / \mathrm{mole}^{1}$ & $\Delta_{\mathrm{f}} \mathrm{H}_{(298)} \mathrm{kcal} \mathrm{mol}^{-1}$ & Error kcal mol $^{-1}$ \\
\hline $\mathrm{CH}_{2} \mathrm{FCHFO} \bullet+\mathrm{CH}_{3} \mathrm{CH}_{3}=\mathrm{CH}_{3} \mathrm{CH}_{2} \mathrm{O} \bullet+\mathrm{CH}_{3} \mathrm{CHF}_{2}$ & & & & \\
\hline$-352.768218-79.717768-154.268107-278.225303$ & -0.00742 & -4.65863 & -99.2 & \pm 1.66 \\
\hline$-20.05-3.01-120.87$ & & & & \\
\hline Reported $\Delta_{\mathrm{f}} \mathrm{H}^{\circ}(298) \mathrm{kcal} \mathrm{mol}^{-1}$ & & & $-99.1 \pm 1.57$ & \\
\hline Standard Deviation over rxns & & & \pm 0.1 & \\
\hline $\mathrm{C} \cdot \mathrm{F}_{2} \mathrm{CH}_{2} \mathrm{OH}+\mathrm{CH}_{4}=\mathrm{CH}_{3} \mathrm{CH}_{2} \mathrm{O} \cdot+\mathrm{CH}_{2} \mathrm{~F}_{2}$ & & & & \\
\hline$-352.773245-40.447961-154.268107-238.938973$ & 0.014126 & 8.864192 & -102.1 & \pm 1.47 \\
\hline $\begin{array}{lll}-17.81 & -3.01 & -108.07\end{array}$ & & & & \\
\hline $\mathrm{C} \cdot \mathrm{F}_{2} \mathrm{CH}_{2} \mathrm{OH}+\mathrm{CH}_{3} \mathrm{CH}_{3}=\mathrm{CH}_{3} \mathrm{CH}_{2} \mathrm{O} \cdot+\mathrm{CH}_{3} \mathrm{CHF}_{2}$ & & & & \\
\hline $\begin{array}{cccc}-352.773245 & -79.717768 & -154.268107 & -278.225303 \\
& -20.05 & -3.01 & -120.87\end{array}$ & -0.0024 & -1.50414 & -102.3 & \pm 1.66 \\
\hline Reported $\Delta_{\mathrm{f}} \mathrm{H}^{\circ}(298) \mathrm{kcal} \mathrm{mol}^{-1}$ & & & $-102.2 \pm 1.57$ & \\
\hline Standard Deviation over rxns & & & \pm 0.2 & \\
\hline $\mathrm{CHF}_{2} \mathrm{CH}_{2} \mathrm{O} \bullet+\mathrm{CH}_{4}=\mathrm{CH}_{3} \mathrm{CH}_{2} \mathrm{O} \cdot+\mathrm{CH}_{2} \mathrm{~F}_{2}$ & & & & \\
\hline$-352.767873-40.447961-154.268107-238.938973$ & 0.008754 & 5.493214 & -98.8 & \pm 1.47 \\
\hline $\begin{array}{lll}-17.81 & -3.01 & -108.07\end{array}$ & & & & \\
\hline $\mathrm{CHF}_{2} \mathrm{CH}_{2} \mathrm{O} \bullet+\mathrm{CH}_{3} \mathrm{CH}_{3}=\mathrm{CH}_{3} \mathrm{CH}_{2} \mathrm{O} \cdot+\mathrm{CH}_{3} \mathrm{CHF}_{2}$ & & & & \\
\hline $\begin{array}{llll}-352.767873 & -79.717768 & -154.268107 & -278.225303\end{array}$ & -0.00777 & -4.87512 & -99.0 & 1.66 \\
\hline $\begin{array}{ccc}-20.05 & -3.01 & -120.87\end{array}$ & & & & \\
\hline Reported $\Delta_{\mathrm{f}} \mathrm{H}^{\mathrm{o}}(298) \mathrm{kcal} \mathrm{mol}^{-1}$ & & & $-98.9 \pm 1.57$ & \\
\hline Standard Deviation over rxns & & & \pm 0.2 & \\
\hline $\mathrm{CH}_{2} \cdot \mathrm{CF}_{2} \mathrm{OH}+\mathrm{CH}_{4}=\mathrm{CH}_{3} \mathrm{O} \cdot+\mathrm{CH}_{3} \mathrm{CHF}_{2}$ & & & & \\
\hline$-352.800434-40.447961-114.989112-278.225303$ & 0.03398 & 21.32276 & -119.2 & \pm 1.71 \\
\hline $\begin{array}{lll}-17.81 & 5.15 & -120.87\end{array}$ & & & & \\
\hline $\begin{array}{l}\mathrm{CH}_{2} \cdot \mathrm{CF}_{2} \mathrm{OH}+\mathrm{CH}_{3} \mathrm{CH}_{3}=\mathrm{CH}_{3} \mathrm{O} \cdot+\mathrm{CH}_{3} \mathrm{CH}_{2} \mathrm{CHF}_{2} \\
-352.800434-79.717768-114.989112-317.498882\end{array}$ & 0.030208 & 18.95579 & -119.6 & \pm 1.77 \\
\hline $\begin{array}{lll}-20.05 & 5.15 & -125.82\end{array}$ & & & & \\
\hline Reported $\Delta_{\mathrm{f}} \mathrm{H}^{\circ}(298) \mathrm{kcal} \mathrm{mol}^{-1}$ & & & $-119.4 \pm 1.74$ & \\
\hline Standard Deviation over rxns & & & \pm 0.2 & \\
\hline $\mathrm{CH}_{3} \mathrm{CF}_{2} \mathrm{O} \cdot+\mathrm{CH}_{4}=\mathrm{CH}_{3} \mathrm{CH}_{2} \mathrm{O} \bullet+\mathrm{CH}_{2} \mathrm{~F}_{2}$ & & & & \\
\hline$-352.786755-40.447961-154.268107-238.938973$ & 0.027636 & 17.34184 & -110.6 & \pm 1.47 \\
\hline-108.07 & & & & \\
\hline $\mathrm{CH}_{3} \mathrm{CF}_{2} \mathrm{O} \bullet+\mathrm{CH}_{3} \mathrm{CH}_{2} \mathrm{CH}_{3}=\mathrm{CH}_{3} \mathrm{CH}_{2} \mathrm{O} \bullet+\mathrm{CH}_{3} \mathrm{CH}_{2} \mathrm{CHF}_{2}$ & & & & \\
\hline$-352.786755-118.990915-154.268107-317.498882$ & 0.010681 & 6.702424 & -110.5 & \pm 1.77 \\
\hline $\begin{array}{lll}-25.02 & 5.15 & -125.82\end{array}$ & & & & \\
\hline Reported $\Delta_{\mathrm{f}} \mathrm{H}^{\mathrm{o}}(298) \mathrm{kcal} \mathrm{mol}^{-1}$ & & & $-110.6 \pm 1.62$ & \\
\hline Standard Deviation over rxns & & & \pm 0.1 & \\
\hline
\end{tabular}

Hartrees, kcal mole ${ }^{-1} *$ SD Standard Deviation $\mathrm{kcal} \mathrm{mol}^{-1}$ Errors reported avg of sum of uncertainties in rxn's reference species

\section{Entropy and Heat Capacity Values}

Internal rotor contributions to calculated entropy and heat capacity at $298-1500 \mathrm{~K}$ were determined using the molecular mass of each molecule, number of optical isomers, symmetry of the molecule, electron degeneracy, moment of inertia, and vibrational frequencies values (table 5). The vibrational frequencies for the calculation of heat capacity and entropy at the $M-062 x / 6-31+g(d, p)$ level of calculation was scaled by a factor of 0.97 . The moment of inertia values is shown in the Supporting Information Table provided. To calculate the contributions of external rotor, vibration and transition to the calculated entropy and heat capacity, the "SMCPS" program is used. It employs the rigid-rotor harmonic oscillator approximation using moment of inertia from optimized

$$
\mathrm{V}(\Phi)=\mathrm{a}_{0}+\Sigma \mathrm{a}_{\mathrm{i}} \cos (\mathrm{i} \Phi)+\Sigma \mathrm{b}_{\mathrm{j}} \cos (\mathrm{j} \Phi)
$$

The coefficients ai and bj are calculated to present the maxima and minima of the torsional potentials with a possibility to shift from the extreme angular positions.

Calculations of heat capacity and standard entropy based on benchmark database and the computational chemistry$$
\mathrm{i}, \mathrm{j}=1-10
$$

structure and frequencies. The "Rotator' program by Lay et al. is used to calculate internal rotor contributions from the corresponding internal rotor torsion frequencies. In this paper, a torsional potential curve presenting a ten-parameter Fourier series function is used to calculate the contribution of internal rotor. Parameters and detailed functions are shown in the Supporting Information Table provided. Rotor [15-18] program is used to calculate thermodynamic functions from hindered rotations with arbitrary potentials.

Calculation of the Hamiltonian matrix of the internal rotor, and subsequent calculation of energy levels by direct diagonalization of the matrix are employed by this technique. Rotational barriers versus dihedral angle is presented as a potential curve. In this paper, the calculated torsional potential at discrete torsional angles

comparison for the $\mathrm{M}-062 \mathrm{x} / 6-31+\mathrm{g}(\mathrm{d}, \mathrm{p})$ calculation method, the vibrational frequencies were scaled by a factor of 0.987 [19]. Potential Energy profiles for mono and di fluorinated ethanol and their related radicals are listed in the Supporting Information Table provided., the solid lines are the fit of 
Fourier series expansion, rotator contribution for barriers entropy and heat capacity. Energies are in kcal mol ${ }^{-1}$. Table 6 . below $7 \mathrm{kcal} \mathrm{mol}^{-1}$ were added to the SMCPS calculated

Table 5. Monofluoro and Difluoro- Ethanol's Ideal Gas phase Entropy and Heat Capacity obtained using M-062x/6-31+g $(d, p)$ level of theory $\left(\right.$ Cal mol $\left.^{-1} K^{-1}\right)$.

\begin{tabular}{|c|c|c|c|c|c|c|c|c|c|}
\hline Species & & $S(298)$ & Cp(298) & Cp(400) & $\mathrm{Cp}(500)$ & Cp(600) & Cp(800) & Cp(1000 & Cp(1500) \\
\hline \multirow{4}{*}{$\mathrm{CH}_{2} \mathrm{FCH}_{2} \mathrm{OH}$} & TVR & 64.74 & 13.23 & 17.12 & 20.72 & 23.75 & 28.18 & 31.11 & 35.06 \\
\hline & Internal Rotor & 3.84 & 3.24 & 3.48 & 3.43 & 3.22 & 2.68 & 2.23 & 1.62 \\
\hline & Total & 2.02 & 3.15 & 3.33 & 3.04 & 2.66 & 2.05 & 1.69 & 1.3 \\
\hline & & 70.6 & 19.62 & 23.94 & 27.2 & 29.62 & 32.91 & 35.03 & 37.98 \\
\hline \multirow{4}{*}{$\mathrm{CH}_{3} \mathrm{CHFOH}$} & TVR & 65.17 & 14.06 & 17.9 & 21.37 & 24.26 & 28.51 & 31.32 & 35.15 \\
\hline & Internal Rotor & 4.48 & 2.13 & 2.09 & 1.95 & 1.8 & 1.56 & 1.4 & 1.19 \\
\hline & Total & 2.67 & 2.17 & 2.21 & 2.18 & 2.09 & 1.89 & 1.71 & 1.4 \\
\hline & & 72.32 & 18.35 & 22.21 & 25.5 & 28.16 & 31.96 & 34.42 & 37.75 \\
\hline \multirow{4}{*}{$\mathrm{C} \cdot \mathrm{HFCH}_{2} \mathrm{OH}$} & TVR & 66.3 & 13.54 & 16.96 & 19.97 & 22.48 & 26.29 & 29.04 & 33.33 \\
\hline & Internal Rotor & 6.19 & 2.31 & 1.91 & 1.69 & 1.54 & 1.36 & 1.25 & 1.13 \\
\hline & Total & 3.09 & 3.16 & 2.52 & 2.04 & 1.74 & 1.41 & 1.25 & 1.11 \\
\hline & & 75.58 & 19.01 & 21.38 & 23.7 & 25.76 & 29.06 & 31.55 & 35.56 \\
\hline \multirow{4}{*}{$\mathrm{CH}_{2} \mathrm{FCH} \cdot \mathrm{OH}$} & TVR & 66.47 & 13.76 & 17.09 & 20.04 & 22.52 & 26.29 & 29.02 & 33.31 \\
\hline & Internal Rotor & 5.72 & 2.34 & 2.22 & 2.09 & 1.95 & 1.73 & 1.56 & 1.32 \\
\hline & Total & 6 & 1.96 & 1.95 & 1.95 & 1.91 & 1.79 & 1.65 & 1.4 \\
\hline & & 78.19 & 18.06 & 21.27 & 24.07 & 26.38 & 29.81 & 32.24 & 36.03 \\
\hline \multirow{3}{*}{$\mathrm{CH}_{2} \mathrm{FCH}_{2} \mathrm{O}$} & TVR & 75.3 & 20.44 & 23.66 & 26.27 & 28.39 & 31.55 & 33.79 & 37.16 \\
\hline & Internal Rotor & 6.08 & 2.2 & 1.96 & 1.7982 & 1.68 & 1.53 & 1.43 & 1.27 \\
\hline & Total & 81.38 & 22.64 & 25.62 & 28.07 & 30.07 & 33.08 & 35.21 & 38.42 \\
\hline \multirow{4}{*}{$\mathrm{CH}_{2} \cdot \mathrm{CHFOH}$} & TVR & 66.89 & 14.8 & 18.21 & 21.07 & 23.4 & 26.9 & 29.44 & 33.49 \\
\hline & Internal Rotor & 4.94 & 1.42 & 1.25 & 1.17 & 1.12 & 1.06 & 1.04 & 1.01 \\
\hline & Total & 2.84 & 1.95 & 2.12 & 2.12 & 2.04 & 1.83 & 1.64 & 1.35 \\
\hline & & 74.67 & 18.17 & 21.58 & 24.35 & 26.55 & 29.79 & 32.11 & 35.86 \\
\hline \multirow{4}{*}{$\mathrm{CH}_{3} \mathrm{CF} \cdot \mathrm{OH}$} & TVR & 66.56 & 13.94 & 17.25 & 20.17 & 22.62 & 26.38 & 29.11 & 33.38 \\
\hline & Internal Rotor & 4.91 & 1.97 & 1.75 & 1.57 & 1.44 & 1.27 & 1.18 & 1.08 \\
\hline & Total & 1.71 & 2.6 & 3.17 & 3.22 & 3.01 & 2.43 & 1.99 & 1.45 \\
\hline & & 73.18 & 18.5 & 22.17 & 24.96 & 27.07 & 30.08 & 32.28 & 35.92 \\
\hline \multirow{3}{*}{$\mathrm{CH}_{3} \mathrm{CHFO}$} & TVR & 66.73 & 14.44 & 18.16 & 21.39 & 24.09 & 28.18 & 31.12 & 35.58 \\
\hline & Internal Rotor & 4.55 & 2.12 & 2.05 & 1.9 & 1.75 & 1.51 & 1.36 & 1.18 \\
\hline & Total & 71.27 & 16.56 & 20.21 & 23.29 & 25.84 & 29.69 & 32.48 & 36.75 \\
\hline \multirow{4}{*}{$\mathrm{CH}_{2} \mathrm{FCHFOH}$} & TVR & 67.46 & 15.41 & 19.51 & 23.12 & 26.07 & 30.23 & 32.83 & 36.15 \\
\hline & Internal Rotor & 0 & 0 & 0 & 0 & 0 & 0 & 0 & 0 \\
\hline & Total & 6.12 & 3.11 & 2.64 & 2.24 & 1.94 & 1.57 & 1.37 & 1.17 \\
\hline & & 73.58 & 18.52 & 22.15 & 25.35 & 28 & 31.79 & 34.2 & 37.31 \\
\hline \multirow{4}{*}{$\mathrm{CF}_{2} \mathrm{HCH}_{2} \mathrm{OH}$} & TVR & 62.94 & 12 & 15.54 & 18.95 & 21.9 & 26.43 & 29.57 & 34.06 \\
\hline & Internal Rotor & 4.98 & 3.36 & 3.2 & 2.97 & 2.72 & 2.26 & 1.93 & 1.48 \\
\hline & Total & 2.23 & 3.21 & 3.17 & 2.81 & 2.44 & 1.91 & 1.6 & 1.27 \\
\hline & & 70.15 & 18.57 & 21.91 & 24.72 & 27.06 & 30.6 & 33.1 & 36.81 \\
\hline \multirow{4}{*}{$\mathrm{CH}_{3} \mathrm{CF}_{2} \mathrm{OH}$} & TVR & 68.41 & 16.53 & 20.74 & 24.23 & 27.01 & 30.87 & 33.29 & 36.37 \\
\hline & Internal Rotor & 4.53 & 2.13 & 2.08 & 1.93 & 1.77 & 1.53 & 1.38 & 1.18 \\
\hline & Total & 1.58 & 2.45 & 3.09 & 3.28 & 3.14 & 2.6 & 2.14 & 1.53 \\
\hline & & 74.52 & 21.11 & 25.91 & 29.44 & 31.93 & 35.01 & 36.8 & 39.08 \\
\hline \multirow{4}{*}{$\mathrm{CH}_{2} \mathrm{FC} \cdot \mathrm{FOH}$} & TVR & 69.58 & 15.15 & 18.76 & 21.84 & 24.36 & 28.05 & 30.59 & 34.38 \\
\hline & Internal Rotor & 6.18 & 3.28 & 2.51 & 2.07 & 1.8 & 1.5 & 1.34 & 1.16 \\
\hline & Total & 3.15 & 1.91 & 1.96 & 1.91 & 1.83 & 1.65 & 1.5 & 1.27 \\
\hline & & 78.9 & 20.34 & 23.22 & 25.82 & 27.99 & 31.19 & 33.43 & 36.81 \\
\hline \multirow{4}{*}{$\mathrm{C} \cdot \mathrm{HFCFHOH}$} & TVR & 69.6 & 15.48 & 19.22 & 22.3 & 24.76 & 28.32 & 30.75 & 34.41 \\
\hline & Internal Rotor & 6.92 & 2.03 & 1.69 & 1.49 & 1.37 & 1.22 & 1.15 & 1.07 \\
\hline & Total & 1.72 & 2.79 & 3.1 & 3.03 & 2.83 & 2.37 & 2.02 & 1.52 \\
\hline & & 78.25 & 20.3 & 24.01 & 26.83 & 28.96 & 31.91 & 33.92 & 37 \\
\hline \multirow{3}{*}{$\mathrm{CH}_{2} \mathrm{FCHFO}$} & TVR & 70.53 & 15.99 & 19.88 & 23.22 & 25.94 & 29.93 & 32.65 & 36.59 \\
\hline & Internal Rotor & 5.48 & 2.92 & 2.79 & 2.58 & 2.37 & 2.01 & 1.76 & 1.4 \\
\hline & Total & 76.01 & 18.91 & 22.67 & 25.8 & 28.31 & 31.94 & 34.41 & 37.99 \\
\hline \multirow{4}{*}{$\mathrm{CHF}_{2} \mathrm{C} \cdot \mathrm{HOH}$} & TVR & 68.27 & 15.46 & 19.18 & 22.25 & 24.71 & 28.28 & 30.72 & 34.4 \\
\hline & Internal Rotor & 6.07 & 2.65 & 2.3 & 2.05 & 1.86 & 1.61 & 1.45 & 1.24 \\
\hline & Total & 1.51 & 2.5 & 3.06 & 3.18 & 3.06 & 2.6 & 2.2 & 1.61 \\
\hline & & 75.85 & 20.61 & 24.54 & 27.48 & 29.63 & 32.49 & 34.37 & 37.25 \\
\hline
\end{tabular}




\begin{tabular}{|c|c|c|c|c|c|c|c|c|c|}
\hline Species & & $S(298)$ & Ср(298) & Cp(400) & Сp(500) & Cp(600) & Cp(800) & Ср(1000 & Cp(1500) \\
\hline \multirow{4}{*}{$\mathrm{C} \cdot \mathrm{F}_{2} \mathrm{CH}_{2} \mathrm{OH}$} & TVR & 69.57 & 15.16 & 18.73 & 21.81 & 24.32 & 28.03 & 30.58 & 34.37 \\
\hline & Internal Rotor & 6.18 & 2.68 & 2.38 & 2.11 & 1.89 & 1.5848 & 1.41 & 1.19 \\
\hline & Total & 3.46 & 2.45 & 2.04 & 1.75 & 1.55 & 1.32 & 1.21 & 1.09 \\
\hline & & 79.21 & 20.29 & 23.15 & 25.66 & 27.76 & 30.94 & 33.19 & 36.65 \\
\hline \multirow{3}{*}{$\mathrm{CHF}_{2} \mathrm{CH}_{2} \mathrm{O}$} & TVR & 70.02 & 15.31 & 18.91 & 22.14 & 24.87 & 29.03 & 31.94 & 36.19 \\
\hline & Internal Rotor & 5.78 & 2.51 & 2.41 & 2.26 & 2.1 & 1.83 & 1.63 & 1.34 \\
\hline & Total & 75.79 & 17.82 & 21.32 & 24.4 & 26.97 & 30.86 & 33.57 & 37.53 \\
\hline \multirow{4}{*}{$\mathrm{CH}_{2} \cdot \mathrm{CF}_{2} \mathrm{OH}$} & TVR & 70.2 & 17.3 & 21.07 & 23.93 & 26.13 & 29.24 & 31.38 & 34.69 \\
\hline & Internal Rotor & 4.97 & 1.37 & 1.23 & 1.16 & 1.11 & 1.06 & 1.04 & 1.01 \\
\hline & Total & 1.78 & 2.81 & 3.33 & 3.25 & 2.9407 & 2.3 & 1.87 & 1.38 \\
\hline & & 76.95 & 21.48 & 25.63 & 28.35 & 27.25 & 32.61 & 34.29 & 37.09 \\
\hline \multirow{4}{*}{$\mathrm{CH}_{3} \mathrm{CF}_{2} \mathrm{O} \bullet$} & TVR & 70.14 & 16.85 & 20.78 & 23.99 & 26.55 & 30.3 & 32.87 & 36.66 \\
\hline & Internal Rotor & 4.84 & 2.08 & 1.89 & 1.7 & 1.54 & 1.35 & 1.23 & 1.11 \\
\hline & Total & 5.78 & 2.51 & 2.41 & 2.26 & 2.1 & 1.83 & 1.63 & 1.34 \\
\hline & & 80.75 & 21.44 & 25.08 & 27.94 & 30.2 & 33.47 & 35.73 & 39.11 \\
\hline
\end{tabular}

Table 6. Bond Dissociation Energy (BDE's) of Monofluoro and Difluoro- Ethanol's.

Bond Energies

\begin{tabular}{|c|c|c|}
\hline Reactions & Bond Dissociation Energy $^{\mathrm{a}}\left(\mathrm{Kcal} \mathrm{mol}^{-1}\right)$ & Error Kcal mol $^{-1}$ \\
\hline & BDE (this study) & \\
\hline \multicolumn{3}{|l|}{$\mathrm{H}-\mathrm{CHFCH}_{2} \mathrm{OH}$} \\
\hline $\begin{array}{ccc}\mathrm{H}-\mathrm{CHFCH}_{2} \mathrm{OH} & =\mathrm{H} \bullet+\cdot \mathrm{CHFCH}_{2} \mathrm{OH} \\
-101.7 \pm 0.1 & 52.1 & -50.7 \pm 0.09\end{array}$ & $103.1 \pm 0.1$ & \pm 0.2 \\
\hline \multicolumn{3}{|l|}{$\mathrm{CH}_{2} \mathrm{FC}-\mathrm{HHOH}$} \\
\hline $\begin{array}{c}\mathrm{CH}_{2} \mathrm{FC}-\mathrm{HHOH}=\mathrm{H} \bullet+\mathrm{CH}_{2} \mathrm{FC} \cdot \mathrm{HOH} \\
-101.7 \pm 0.1 \quad 52.1-56.1 \pm 0.1\end{array}$ & $97.7 \pm 0.1$ & \pm 0.2 \\
\hline \multicolumn{3}{|l|}{$\mathrm{CH}_{2} \mathrm{FCH}_{2} \mathrm{O}-\mathrm{H}$} \\
\hline 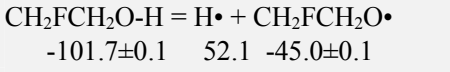 & $108.8 \pm 0.1$ & \pm 0.2 \\
\hline \multicolumn{3}{|l|}{$\mathrm{H}-\mathrm{CH}_{2} \mathrm{CHFOH}$} \\
\hline $\begin{array}{ccc}\mathrm{H}-\mathrm{CH}_{2} \mathrm{CHFOH} & =\mathrm{H} \bullet+\cdot \mathrm{CH}_{2} \mathrm{CHFOH} \\
-113.5 \pm 0.2 & 52.1 & -59.6 \pm 0.1\end{array}$ & $106.0 \pm 0.15$ & \pm 0.3 \\
\hline \multicolumn{3}{|l|}{$\mathrm{CH}_{3} \mathrm{C}-\mathrm{HFOH}$} \\
\hline $\mathrm{H}-\mathrm{CH}_{2} \mathrm{CHFOH}=\mathrm{H} \bullet+\mathrm{CH}_{3} \mathrm{C} \cdot \mathrm{FOH}$ & $97.6 \pm 0.2$ & \pm 0.4 \\
\hline \multicolumn{3}{|l|}{$\mathrm{CH}_{3} \mathrm{CHFO}-\mathrm{H}$} \\
\hline $\mathrm{CH}_{3} \mathrm{CHFO}-\mathrm{H}=\mathrm{H} \bullet+\mathrm{CH}_{3} \mathrm{CHFO} \bullet$ & $106.8 \pm 0.2$ & \pm 0.4 \\
\hline \multicolumn{3}{|l|}{$\mathrm{H}$-CHFCHFOH } \\
\hline $\mathrm{H}-\mathrm{CHFCHFOH}=\mathrm{H} \bullet+\cdot \mathrm{CHFCHFOH}$ & $101.1 \pm 0.4$ & \pm 0.8 \\
\hline \multirow{2}{*}{$\begin{array}{l}\mathrm{CH}_{2} \mathrm{FC}-\mathrm{HFOH} \\
\mathrm{CH}_{2} \mathrm{FC}-\mathrm{HFOH}=\mathrm{H} \bullet+\mathrm{CH}_{2} \mathrm{FC} \cdot \mathrm{FOH}\end{array}$} & $102.6 \pm 0.35$ & \pm 0.7 \\
\hline & \multicolumn{2}{|c|}{$\begin{array}{ccc}\mathrm{CH}_{2} \mathrm{FC}-\mathrm{H} U \mathrm{H} & =\mathrm{H}^{\bullet}+\mathrm{CH}_{2} \mathrm{FC} \cdot \mathrm{FOH} \\
-154.6 \pm 0.6 & 52.1 & -104.1 \pm 0.1\end{array}$} \\
\hline $\mathrm{CH}_{2}$ FCHFO-H & $107.6 \pm 0.35$ & \\
\hline $\begin{array}{c}\mathrm{CH}_{2} \mathrm{FCHFO}-\mathrm{H}=\mathrm{H} \bullet+\mathrm{CH}_{2} \mathrm{FCHFO} \bullet \\
154.6 \pm 0.652 .1-99.1 \pm 0.1\end{array}$ & $105.1 \pm 0.15$ & \pm 0.3 \\
\hline \multicolumn{3}{|l|}{$\mathrm{CF}_{2}-\mathrm{HCH}_{2} \mathrm{OH}$} \\
\hline $\mathrm{CF}_{2}-\mathrm{HCH}_{2} \mathrm{OH}=\mathrm{H} \bullet+\cdot \mathrm{CF}_{2} \mathrm{CH}_{2} \mathrm{OH}$ & $105.0 \pm 0.15$ & \pm 0.3 \\
\hline \multicolumn{3}{|l|}{$\mathrm{CF}_{2} \mathrm{HCH}-\mathrm{HOH}$} \\
\hline $\begin{array}{ccc}\mathrm{CF}_{2} \mathrm{HCHOH}-\mathrm{H} & =\mathrm{H} \bullet+\mathrm{CF}_{2} \mathrm{HC} \cdot \mathrm{OH} \\
-155.3 \pm 0.1 & 52.1 & -102.4 \pm 0.2\end{array}$ & & \\
\hline \multicolumn{3}{|l|}{$\mathrm{CF}_{2} \mathrm{HCH}_{2} \mathrm{O}-\mathrm{H}$} \\
\hline $\begin{array}{c}\mathrm{CF}_{2} \mathrm{HCH}_{2} \mathrm{O}-\mathrm{H}=\mathrm{H} \bullet+\mathrm{CF}_{2} \mathrm{HCH}_{2} \mathrm{O} \bullet \\
-155.3 \pm 0.1 \\
52.1 \quad-98.9 \pm 0.2\end{array}$ & $108.5 \pm 0.15$ & \pm 0.3 \\
\hline \multicolumn{3}{|l|}{$\mathrm{H}-\mathrm{CH}_{2} \mathrm{CF}_{2} \mathrm{OH}$} \\
\hline $\mathrm{H}-\mathrm{CH}_{2} \mathrm{CF}_{2} \mathrm{OH}=\mathrm{H} \bullet+\cdot \mathrm{CH}_{2} \mathrm{CF}_{2} \mathrm{OH}$ & $107.2 \pm 0.15$ & \pm 0.3 \\
\hline \multicolumn{3}{|l|}{$\mathrm{CH}_{3} \mathrm{CF}_{2} \mathrm{O}-\mathrm{H}$} \\
\hline $\begin{array}{c}\mathrm{CH}_{3} \mathrm{CF}_{2} \mathrm{O}-\mathrm{H}=\mathrm{H} \bullet+\mathrm{CH}_{3} \mathrm{CF}_{2} \mathrm{O} \bullet \\
-174.5 \pm 0.1 \quad 52.1 \quad-110.6 \pm 0.1\end{array}$ & $116.0 \pm 0.1$ & \pm 0.2 \\
\hline
\end{tabular}

a standard deviation among the work reactions. 


\section{Conclusions}

Thermodynamic properties of 19 mono and di-fluoro ethanols and their related radicals are calculated using the $\mathrm{ab}$ initio and Global-hybrid meta-GGA density function methods. Isodesmic work reactions are employed for cancellation of calculation errors. Multiple work reactions are utilized to calculate standard enthalpy of formation at the Gaussian M06-2X calculation level. Optimized geometries and frequencies are used to determine entropy and heat capacity with $\mathrm{M} 06-2 \mathrm{x} / 6-31+\mathrm{g}(\mathrm{d}, \mathrm{p})$ level of calculation. Intermolecular torsion potential curves at the $\mathrm{M}-06-2 \mathrm{x} / 6-31+\mathrm{g}(\mathrm{d}, \mathrm{p})$ level of calculation are used to calculate hindered internal rotation contributions to heat capacity and entropy with a correction to the calculated heat capacity and entropy. The Thermochemical properties: Entropy, Heat Capacities at $(298-1500 \mathrm{~K})$, Standard Enthalpy of formation (298K), and the $\mathrm{C}-\mathrm{F}$ and $\mathrm{C}-\mathrm{H}$ Bond Dissociation Energies (BDEs) for Mono and Difluorinated Ethanols and Radicals: $\mathrm{CH}_{3}-\mathrm{xCHFxOH}$, $\mathrm{CH}_{3} \mathrm{CH}_{2}-\mathrm{xFxOH}$ have been calculated. The $\mathrm{C}-\mathrm{H}$ bond energies range from 102.2 to $107.2 \mathrm{Kcal} \mathrm{mol}^{-1}$ on the methyl carbons, and from 97.3 to $105.2 \mathrm{Kcal} \mathrm{mol}^{-1}$ on the secondary ethyl carbons. The calculated values for $\mathrm{C}-\mathrm{H}$ bond energies for fluorinated methyl carbons are higher than those of the fluorinated ethyl carbons. Calculated values for the $\mathrm{O}-\mathrm{H}$ bond energies for 2-fluoroethanol are higher than those of $\mathrm{O}-\mathrm{H}$ bond energies for 1-fluoroethanol and an intermediate calculated value for $\mathrm{O}-\mathrm{H}$ bond energies for 1, 2-difluoroethanol. Introducing a fluorine atom to either methyl or ethyl carbon increases $\mathrm{O}-\mathrm{H}$ bond energy [20].

\section{Supporting Information}

Supporting information is available, Cartesian Coordinates; Z-matrixes, vibration frequencies, moments of inertia, the method of standard deviation, Optimized Geometries, and C$\mathrm{C}$ and $\mathrm{C}-\mathrm{O}$ internal rotors potential energy profile for target fluorinated ethanol and their related radicals are included.

\section{Acknowledgements}

We acknowledge the NJIT Advanced Research Computing Services for significant help in providing the computer calculation software.

\section{References}

[1] Wallington, T. J.; Schneider, W. F.; Worsnop, D. R.; Nielsen, O. J.; Sehested, J.; Debruyn, W. J.; Shorter, J. A. The Environmental Impact of CFC Replacements HFCs and HCFCs. Environ. Sci. Technol. 1994, 28, 320A-326A.

[2] Wang H., Castillo A., Bozzelli J. W., Thermochemical properties enthalpy, entropy, and heat capacity of C1-4 fluorinated hydrocarbons. J. Phys. Chem. 2015.
[3] Schneider, W. F.; Wallington, T. J. Ab Initio Investigation of the Heats of Formation of Several Trifluoromethyl Compounds. J. Phys. Chem. 1993, 97, 12783-12788.

[4] Wang, H.; Castillo, Á.; Bozzelli, J. W. Thermochemical Properties Enthalpy, Entropy, and Heat Capacity of C1-C4 Fluorinated Hydrocarbons: Fluorocarbon Group Additivity. J. Phys. Chem. A 2015, 119, 8202-8215.

[5] El-Taher, S. Ab Initio and DFT Investigation of Fluorinated Methyl Hydroperoxides: Structures, Rotational Barriers, and Thermo chemical Properties. J. Fluorine Chem. 2006, 127, 54-62.

[6] Wang H., Bozzelli J. W., Thermochemical properties and Bond Dissociation Energy for Fluorinated Methanol and fluorinated methyl hydroperoxides,. J. Phys. Chem. 2016.

[7] Ruscic, B., Active Thermochemical Tables: Sequential Bond Dissociation Enthalpy of Methane, Ethane, and Methanol and related Thermochemistry. J. Phys. Chem. A 2015, 119, 78107837.

[8] Burke, S. M.; Simmie, J. M.; Curran, H. J. Critical Evaluation of Thermochemical Properties of $\mathrm{C} 1-\mathrm{C} 4$ Species: Updated Group Contributions to Estimate Thermochemical Properties. J. Phys. Chem. Ref. Data 2015, 44, 013101.

[9] Chase, M. W. J. NIST-JANAF Thermochemical Tables. J. Phys. Chem. Ref. Data. 1998, Monograph 9, 1-1951.

[10] Luo, X.; Fleming, P. R.; Rizzo, T. R. Vibrational Overtone Spectroscopy of the $4 v \mathrm{OH}+v \mathrm{OH}^{\prime}$ Combination Level of $\mathrm{HOOH}$ via Sequential Local Mode -local Mode Excitation. J. Chem. Phys. 1992, 96, 5659-5667.

[11] Bodi, A.; Kercher, J. P.; Bond, C.; Meteesatien, P.; Sztáray, B.; Baer, T. Photoion Photoelectron Coincidence Spectroscopy of Primary Amines RCH $2 \mathrm{NH}_{2}\left(\mathrm{R}=\mathrm{H}, \mathrm{CH}_{3}, \mathrm{C} 2 \mathrm{H}_{5}, \mathrm{C} 3 \mathrm{H}_{7}\right.$, i$\mathrm{C}_{3} \mathrm{H}_{7}$ ): Alkylamine and Alkyl Radical Heats of Formation by Isodesmic Reaction Networks. J. Phys. Chem. A 2006, 110, 13425-13433.

[12] Wang, H.; Bozzelli, J. W. Thermochemical Properties ( $\Delta \mathrm{fH}$ (298 K), S (298 K), C p(T)) and Bond Dissociation Energies for C1-C4 Normal Hydroperoxides and Peroxy Radicals. J. Chem. Eng. Data 2016, 61, 1836-1849.

[13] Csontos, J.; Rolik, Z.; Das, S.; Kállay, M. High-Accuracy Thermochemistry of Atmospherically Important Fluorinated and Chlorinated Methane Derivatives. J. Phys. Chem. A 2010, $114,13093-13103$.

[14] Math is Fun Advanced, 2017, Standard Deviation and Variance, 12/2019,

\{https://www.mathsisfun.com/data/standard-deviation.html\}

[15] NIST Computational Chemistry Comparison and Benchmark Database. NIST Standard Reference Database Number, Release 16a; Johnson, R. D., III, Ed.; NIST: Gaithersburg, MD, 2013.

[16] Sheng, C. Elementary, Pressure Dependent Model for Combustion of $\mathrm{C} 1, \quad \mathrm{C} 2$ and Nitrogen Containing Hydrocarbons: Operation of A Pilot Scale Incinerator and Model Comparison. Ph. D. dissertation; New Jersey Institute of Technology, 2002.

[17] Becke, A. D. Density-functional Thermochemistry. III. The Role of Exact Exchange. J. Chem. Phys. 1993, 98, 5648-5652. 
[18] Lay, T. H.; Krasnoperov, L. N.; Venanzi, C. A.; Bozzelli, J. W.; Shokhirev, N. V. Ab Initio Study of $\alpha$-Chlorinated Ethyl Hydro510peroxides $\mathrm{CH}_{3} \mathrm{CH}_{2} \mathrm{OOH}, \mathrm{CH}_{3} \mathrm{CHClOOH}$, and $\mathrm{CH}_{3} \mathrm{CCl}_{2} \mathrm{OOH}$ : Conformational Analysis, Internal Rotation Barriers, Vibrational Frequencies, and Thermodynamic Properties. J. Phys. Chem. 1996, 513100, 8240-8249.

[19] NIST Computational Chemsitry Comparison and Benchmark Database, NIST Standard Reference Database Number 101, Release 16a; Johnson, R. D. III, Ed.; NIST: Gaithersburg, MD, http://cccbdb. nist. gov (accessed Aug 2013).
[20] Myrna H. M., Minh T. N., David A D, Minh T. N., Theoretical Prediction of the Heats of Formation of $\mathrm{C}_{2} \mathrm{H}_{5} \mathrm{O}^{*}$ Radicals Derived From Ethanol and of the Kinetics of beta-CC Scission in the Ethoxy Radical, J. Phys. Chem. A 2007, 111, $1,113-126$

[21] Density Functional theory, 2019, Density Functionals from the Truhlar Group, University of Minnesota, 11/25/2019, https://comp.chem.umn.edu/info/DFT.htm 